\title{
Clinical significance and management of right heart thrombi: more questions than answers
}

\author{
David Jiménez ${ }^{1}$ and Roger D. Yusen ${ }^{2}$
}

Affiliations: ${ }^{1}$ Respiratory Dept, Ramón y Cajal Hospital and Instituto Ramón y Cajal de Investigación Sanitaria IRYCIS, Madrid, Spain. ${ }^{2}$ Divisions of Pulmonary and Critical Care Medicine and General Medical Sciences, Washington University School of Medicine, St. Louis, MI, USA.

Correspondence: David Jiménez, Respiratory Department and Medicine Department, Ramón y Cajal Hospital, IRYCIS and Alcalá de Henares University 28034 Madrid, Spain. E-mail: djimenez.hrcagmail.com

@ERSpublications

Haemodynamic status has the strongest prognostic implications for short-term mortality in patients with PE http://ow.ly/VEARC

Pulmonary embolism (PE) remains one of the leading causes of cardiovascular morbidity and mortality [1]. Acute PE has a spectrum of clinical syndromes and varying clinical outcomes [2,3]. Estimation of patient prognosis helps to prioritise appropriate management strategies. Risk stratification of patients with PE may identify patients at high risk of early PE-related death who may benefit from escalated surveillance or therapy $[4,5]$. Alternatively, clinicians may consider patients deemed at low risk for early complications (i.e. all-cause mortality, recurrent venous thromboembolism (VTE), and major bleeding) as candidates for partial or complete outpatient PE treatment [6]. According to the recent European Society of Cardiology (ESC) guidelines [7], risk assessment of patients diagnosed with PE should rely on the sequential application of a validated clinical score (i.e. pulmonary embolism severity index (PESI) or simplified PESI (sPESI)), imaging testing assessing right ventricle (RV) function (i.e. echocardiography or computed tomographic pulmonary angiography), and cardiac biomarkers (i.e. cardiac troponins). Though the guidelines state that patients who have acute PE associated with right heart thrombi (RHT) have a potentially life-threatening condition, the authors did not formally incorporate the presence or absence of RHT into their PE prognostication algorithm.

RHT typically represent mobilised deep vein thromboses that have become lodged temporarily in the right atrium or RV $[8,9]$. Though the increased use of two-dimensional echocardiography for risk stratification of PE patients has led to increased detection of RHT, the incidence of RHT remains unknown. In patients with acute PE, RHT pose an unclear risk of mortality [10]. Furthermore, the lack of prospective randomised trials comparing anticoagulant, thrombolytic, catheter-based, and surgical therapies leaves us without guidance regarding optimal management of RHT.

In this issue of the European Respiratory Journal, Koc et al. [11] report the results of an intriguing study aimed at determining the prognostic significance of RHT and their potential influence on treatment selection. The authors used data from a prospective, multicentre, international Right Heart Thrombi European Registry (RiHTER) in order to assess the independent association between RHT characteristics (i.e. size and mobility) and 30-day all-cause mortality. Furthermore, the study compared the outcomes of patients diagnosed with RHT with a subset of patients from a different database who did not have RHT but were otherwise matched according to relevant clinical factors (e.g. age, sex, right to left ventricular diameter ratio, and heart rate). The clinical prognostic scores (i.e. sPESI [12] and shock index [13]) of the patients predicted mortality, while the RHT characteristics did not. However, patients with RV dysfunction and RHT had an increased mortality compared to those with RV dysfunction without RHT. The authors concluded that 30-day mortality in patients with RHT was related to the haemodynamic consequences,

Received: Nov 252015 | Accepted: Nov 302015

Conflict of interest: None declared.

Copyright OERS 2016 
and not to the RHT characteristics themselves. Patients with RV dysfunction and concomitant RHT had worse prognosis than patients with RV dysfunction without RHT.

This RiHTER study had some methodological limitations. All patients who were diagnosed with acute symptomatic PE at the participating sites during the study period did not undergo echocardiography, possibly creating a selection bias. The proportions of patients within each ESC risk class (low risk 17.3\%, intermediate risk $60.1 \%$, and high risk 22.5\%) significantly differed from previous studies [14], further suggesting selection bias. The small number of patients within each ESC risk class, within the study, limited the precision of their estimates and may have created limitations for the predictive models. Though the authors did attempt to reduce the impact of any imbalance in patient characteristics between the comparison groups by using a propensity-based analysis, showing how well the matching occurred would have provided more support of the validity of the results.

The RiHTER study of RHT provides further evidence of the validity of the ESC 2014 prognostic algorithm that assesses risk for all-cause mortality and PE-related complications that occur within 30 days of acute symptomatic $\mathrm{PE}$ diagnosis, despite the presence or absence or RHT. The study validates the presence of PE-associated arterial hypotension or shock as a determinant of high-risk PE. The study also suggests that low-risk patients (according to a well validated clinical prediction rule), who could potentially undergo an abbreviated hospital stay or complete outpatient PE therapy, might not need to undergo echocardiography to confirm or exclude RHT. In a previous study that enrolled 591 normotensive patients with echocardiographic RV dysfunction, the detection of residual deep venous thrombosis by complete compression ultrasound suggested that an elevated risk for recurrent PE existed [15]. A PE recurrence could destabilise these marginally stable patients. The findings from the RiHTER registry support this concept of risk associated with clot burden, since the presence of RHT in patients with PE and RV dysfunction significantly increased the 30-day mortality from $7 \%$ to $16 \%$.

Based on their study, Koć et al. [11] posed the question of whether early thrombolysis should complement the treatment of PE patients with RV dysfunction and concomitant RHT. The stage seems set for clinical trials to address this controversy. Until results from other studies provide more evidence-based guidance, patients with RHT, especially those deemed at high risk for adverse outcomes, should undergo cautious monitoring and individualised treatment. A multidisciplinary Pulmonary Embolism Response Team (PERT) [16] that rapidly evaluates patients, formulates a treatment plan, and mobilises the necessary resources may assist with this endeavour.

\section{References}

1 Jiménez D, de Miguel-Díez J, Guijarro R, et al. Trends in the management and outcomes of acute pulmonary embolism: analysis from the RIETE registry. J Am Coll Cardiol 2016; 67: 162-170.

2 Laporte S, Mismetti P, Décousus H, et al. Clinical predictors for fatal pulmonary embolism in 15,520 patients with venous thromboembolism: findings from the Registro Informatizado de la Enfermedad TromboEmbolica venosa (RIETE) Registry. Circulation 2008; 117: 1711-1716.

3 Goldhaber SZ, Visani L, De Rosa M. Acute pulmonary embolism: clinical outcomes in the International Cooperative Pulmonary Embolism Registry (ICOPER). Lancet 1999; 353: 1386-1389.

4 Bova C, Sanchez O, Prandoni P, et al. Identification of intermediate-risk patients with acute symptomatic pulmonary embolism. Eur Respir J 2014; 44: 694-703.

5 Kearon C, Akl EA, Comerota AJ, et al. Antithrombotic therapy for VTE disease: antithrombotic therapy and prevention of Ttrombosis, 9th ed: American College of Chest Physicians. Evidence-based clinical practice guidelines. Chest 2012; 141: Suppl., e419S-e494S.

6 Jiménez D, Kopecna D, Tapson V, et al. Derivation and validation of multimarker prognostication for normotensive patients with acute symptomatic pulmonary embolism. Am J Respir Crit Care Med 2014; 189: 718-726.

7 Konstantinides SV, Torbicki A, Agnelli G, et al. 2014 ESC Guidelines on the diagnosis and management of acute pulmonary embolism. Eur Heart J 2014; 35: 3033-3073.

8 The European Cooperative Study on the clinical significance of right heart thrombi. Eur Heart J 1989; 10: 1046-1059.

9 Chartier L, Béra J, Delomez M, et al. Free-floating thrombi in the right heart: diagnosis, management, and prognostic indexes in 38 consecutive patients. Circulation 1999; 99: 2779-2783.

10 Rose PS, Punjabi NM, Pearse DB. Treatment of right heart thromboemboli. Chest 2002; 121: 806-814.

11 Koć M, Kostrubiec M, Elikowski W, et al. Outcome of patients with right heart thrombi: the Right Heart Thrombi European Registry. Eur Respir J 2016; 47: 869-875.

12 Jiménez D, Aujesky D, Moores L, et al. Simplification of the pulmonary embolism severity index for prognostication in patients with acute symptomatic pulmonary embolism. Arch Intern Med 2010; 170: 1383-1389.

13 Sam A, Sánchez D, Gómez V, et al. The shock index and the simplified PESI for identification of low-risk patients with acute pulmonary embolism. Eur Respir J 2011; 37: 762-766.

14 Becattini C, Casazza F, Forgione C, et al. Acute pulmonary embolism: external validation of an integrated risk stratification model. Chest 2013; 144: 1539-1545.

15 Jiménez D, Aujesky D, Moores L, et al. Combinations of prognostic tools for identification of high-risk normotensive patients with acute symptomatic pulmonary embolism. Thorax 2011; 66: 75-81.

16 Provias T, Dudzinski DM, Jaff MR, et al. The Massachusetts General Hospital Pulmonary Embolism Response Team (MGH PERT): creation of a multidisciplinary program to improve care of patients with massive and submassive pulmonary embolism. Hosp Pract (1995) 2014; 42: 31-37. 